\title{
Research Square \\ The Effect of Childhood Trauma on Personality in Unaffected First-Degree Relatives of Major Depressive Disorder
}

\section{Chuanyue Wang}

Capital Medical University

\section{Zhen Mao}

Capital Medical University

Feng Li

Capital Medical University

\section{Fan He}

Capital Medical University

Fang Dong

Capital Medical University

\section{Xin $\mathrm{Ma}$}

Capital Medical University

\section{Qijing Bo}

Capital Medical University

\section{Yujie Wen}

Capital Medical University

\section{Wenpeng Hou}

Capital Medical University

Xianbin Li ( $\sim$ xianbinli@ccmu.edu.co0m)

Capital Medical University

\section{Yilang Tang}

Capital Medical University

\section{Research Article}

Keywords: childhood trauma, personality, major depressive disorder, first-degree relatives

Posted Date: October 1st, 2021

DOl: https://doi.org/10.21203/rs.3.rs-844647/v1 
License: (c) (i) This work is licensed under a Creative Commons Attribution 4.0 International License. Read Full License 
3 DEPRESSIVE DISORDER

4 Yujie Wen ${ }^{1,2}$, Qijing Bo ${ }^{1,2}$, Wenpeng Hou ${ }^{1}$, Zhen Mao ${ }^{1,2}$, Feng Li $^{1,2}$, Fan He ${ }^{1,2}$,

5 Fang Dong ${ }^{1,2}$, Xin Ma ${ }^{1,2}$, Yilang Tang ${ }^{1,3}$, Xianbin $\mathrm{Li}^{1,2}, *$, Chuanyue Wang ${ }^{1,2}$

6 Author affiliations:

7 1. The National Clinical Research Center for Mental Disorders \& Beijing Key

8 Laboratory of Mental Disorders, Beijing Anding Hospital, Capital Medical University,

$9 \quad$ Beijing 100088, China

2. Advanced Innovation Center for Human Brain Protection, Capital Medical 11 University, Beijing, China.

12 3. Department of Psychiatry and Behavioral Sciences, Emory University School of 13 Medicine, Atlanta, GA, USA.

\section{Declaration:}

Ethics approval and consent to participate: the protocol was approved by the ethics committee (IRB) of Beijing Anding Hospital, Capital Medical University. We confirm that all methods were performed in accordance with the relevant guidelines and regulations.

Consent for publication: this paper has not been published elsewhere in whole or in part. All authors have read and approved the content and agree to submit it for

21 consideration for publication in your journal. 

generated or analyzed during this study are included in this article.

Competing interests: there are no ethical/legal conflicts involved in the article.

Funding: this work was supported by the National Science Foundation of China (81971287, 81601169, 81471365), the Beijing Municipal Administration of Hospitals Clinical Medicine Development of Special Funding Support (ZYLX201807, XLMX201807), Capital's Funds for Health Improvement and Research (2018-2-2123) and Natural Science Foundation of Capital Medical University (PYZ20001).

Authors' contributions: Xianbin Li, Xin Ma, Chuanyue Wang contributed to the conception of the study; Qijing Bo, Zhen Mao, Feng Li, Fan He, Fang Dong performed the experiment; Wenpeng Hou contributed significantly to analysis and manuscript preparation; Yujie Wen, Qijing Bo performed the data analyses and wrote the manuscript; Yilang Tang helped perform the analysis with constructive discussions.

Acknowledgements: The authors gratefully acknowledge the financial supports by the National Science Foundation of China as well as other foundations.

\section{Author information:}

Chuanyue Wang (wcymanu@163.com)

Zhen Mao (maozhen2015@126.com)

Feng Li (lifengweiyi023@126.com)

Fan He (hf981207@163.com)

Fang Dong (13164295608@163.com)

Xin Ma (maxinanding@vip.163.com)

Qijing Bo (bqj718@163.com) 
Yujie Wen (273600342@qq.com)

Wenpeng Hou (wenpenghou@ccmu.edu.com)

Yilang Tang (ytang5 @emory.edu)

Corresponding author:

Xianbin Li, MD, PhD

Beijing Anding Hospital

Capital Medical University

No.5 Ankang Lane, Dewai Avenue, Xicheng District

Beijing, P. R. China 100088

Telephone: +86-10-58303119

Fax: +86-10-58303119

E-mail: xianbinli@ccmu.edu.com

Running title: The effect of CT on personality in unaffected FDR

Significant Outcomes: In FDRs, CT was associated with a higher score on neuroticism, psychoticism and lie, with the association with neuroticism the strongest. Besides, in terms of subtype, emotional neglect was the one to be found to have the strongest association with neuroticism.

Limitations: Data on CT were obtained using the childhood trauma QuestionnaireShort Form (CTQ-SF), which was a retrospective self-report questionnaire without independent authentication. Accordingly, the validity of reports might be affected by possible memory biases of the patients or excessive sensitivities to some CT experiences. Second, only a few risk factors were included, and the generalizability of 
68 sample size of FDRs of patients with MDD, findings should be considered to be the 69 preliminary.

70 First author : Yujie Wen ; Co first author: Qijing Bo

71 *Corresponding author:

72 Xianbin $\mathrm{Li}, \mathrm{MD}, \mathrm{PhD}$

73 Beijing Anding Hospital

74 Capital Medical University

75 No.5 Ankang Lane, Dewai Avenue, Xicheng District

$76 \quad$ Beijing, P. R. China 100088

77 Telephone: +86-10-58303119

78 Fax: +86-10-58303119

79 E-mail: xianbinli@ccmu.edu.com

80

81

82

83

84

85

86

87

88 


\section{Abstract:}

Objectives: This study is aimed to examine the potential association between childhood trauma $(\mathrm{CT})$ and personality traits among unaffected first-degree relatives (FDR, children or siblings of patients) of patients with major depressive disorder (MDD).

Methods: The study consists of three groups: total 85 patients with MDD, 35 FDRs and 89 healthy control individuals (HC). The Childhood Trauma Questionnaire (CTQ) was used to assess childhood trauma and the Eysenck Personality Questionnaire used to assess personality traits.

Results: By comparison made in personality traits, MDD patients exhibits some significant disparities to FDR and $\mathrm{HC}(\mathrm{p}<0.05$ for extraversion, neuroticism and psychoticism). Nevertheless, no significant difference was found between HC and FDR. In FDR group, patients with CT scored noticeably higher for neuroticism (N) compared with those without $\mathrm{CT}(\mathrm{F}=3.246, \mathrm{p}=0.046)$. CT was associated with $\mathrm{N}$, psychoticism $(\mathrm{P})$ and Lie (L), and it was associated with $\mathrm{N}$ more closely $(\mathrm{r}=0.290-0.452, \mathrm{p}<0.05$ for all). Significantly positive correlations were found between $\mathrm{N}$ and sexual abuse (SA), emotional neglect $(\mathrm{EN})$, physical neglect $(\mathrm{PN})$, and CTQ total $(\mathrm{r}=0.344-0.452, \mathrm{p}<0.05)$; $\mathrm{P}$ and CTQ $(\mathrm{r}=0.336, \mathrm{p}<0.05)$; and significant negative correlations between $\mathrm{L}$ and $\mathrm{EN}$, CTQ $(r=-0.446-0.375, p<0.05)$. EN contributed to a probability of $N, P\left(R^{2}=0.155\right.$ $0.214, \mathrm{~F}=6.066-9.010, \mathrm{p}=0.005-0.019)$ as well as a probability of $\mathrm{L}\left(\mathrm{R}^{2}=0.199, \mathrm{~F}=8.211\right.$, $\mathrm{p}=0.007)$.

Conclusion: $\mathrm{CT}$ was associated with $\mathrm{N}, \mathrm{P}$ and $\mathrm{L}$, with a closer relation to $\mathrm{N}$ in unaffected FDR. Besides, the type of $\mathrm{CT}$, the most relevant to $\mathrm{N}$, was discovered to be EN. Thus, FDR of MDD who experienced CT should be prioritized.

Key words: childhood trauma; personality; major depressive disorder; first-degree relatives 


\section{Introduction}

Numerous studies have supported the correlation between personality and the presence of major depressive disorder (MDD) [1, 2]. Compared with non-affected controls, patients with MDD often have higher scores on neuroticism [3], and the score tend to decrease when patients show improvement of achieve remission [4]. Besides, a higher score on neuroticism has been found to be predictive of poorer outcome in patients with MDD [5-7]. Population-based twin studies showed that the association between neuroticism and MDD may be in part due to shared genetic factors, and they found the genetic correlation with neuroticism was $0.46-0.47$ based on their study of 20,692 same-sex twin pairs in Sweden [8]. Personality traits appear to be vital for the onset and course of depression in many ways $[9,10]$. However, the findings on whether first-degree relatives (FDR) exhibited different personality traits than unaffected controls are inconsistent $[11,12]$.

Studies have found personality traits, especially maladaptive traits of adults are associated with CT, including emotional abuse (EA), physical abuse (PA), sexual abuse (SA), emotional neglect (EN) and physical neglect (PN) in the general population [1315]. Childhood trauma (CT) has been confirmed as a risk factor for major depressive disorder. CT has been found to be associated with the severity in patients with MDD [16]. A meta-analysis of prospective cohort studies found that CT was significantly associated with risk of depression in adults [17]. Moreover, CT has also been found to be poorer clinical course, earlier age of onset $[18,19]$, episode persistence and recurrence [20] in patients with MDD. Recent studies found personality may mediate the effects of childhood abuse on severity of depressive symptoms in patients with MDD [21, 22] as well as the general population [23-26]. Neuroticism, extraversion, 
hopelessness and external locus of control have been reported to mediate the relationship between CT and 4-year remission of depressive and anxiety disorders [27].

These studies all suggest that there is a complex association between CT, personality, severity of depressive symptoms in the general adults and patients with MDD. However, no studies have examined the association between CT and personality traits in FDR of patients with MDD. Therefore, this study was designed to answer the questions above. The Childhood Trauma Questionnaire-Short Form (CTQ-SF) was used to assess the characteristic of childhood trauma and the Eysenck Personality Questionnaire (EPQ) was used to assess personality traits. We started an assessment of the potential association between $\mathrm{CT}$ and personality traits in FDR of patients with MDD. The hypotheses were: (1) FDRs have more prominent maladaptive personality traits comparing with $\mathrm{HC},(2) \mathrm{CT}$ is associated with certain personality traits in FDR.

\section{Methods}

\section{Participants}

This was a cross-sectional study conducted at Beijing Anding Hospital, Capital Medical University, Beijing, China. The protocol was approved by the ethics committee (IRB) of Beijing Anding Hospital, Capital Medical University.

All participants were recruited from September 2014 to September 2016. The study sample consisted of three groups: patients with MDD ( $n=85)$; FDRs of MDD ( $n=35)$; and healthy control $(\mathrm{HC}, \mathrm{n}=89)$. The Structured Clinical Interview for DSM-IV Axis I disorders-Patient Edition (SCID-I/P) was used for diagnosis and the diagnosis of MDD was made according to the Diagnostic and Statistical Manual of Mental Disorders, Fourth Edition (DSM-IV) [28]. The FDR and HC recruited here had no current Axis I diagnose of psychiatric or cognitive disorders. 
The inclusion criteria for the study were as follows: (1) aged between 16 and 55;

163

(2) received more than 9 years of formal education; (3) was able to understand and willing to sign an informed consent. Participants with any of the following were excluded: (1) with current diagnosis of substance abuse or dependence (did not including nicotine); (2) with an unstable, major chronic medical or neurological condition; (3) had received electric convulsive therapy in the recent 3 months.

Based on the results of Childhood Trauma Questionnaire-Short Form (CTQ-SF): The CT positive subgroup consisted of individuals who had one or more scaled scores reaching moderate or severe levels of trauma on the CTQ-SF, participants in three groups (HC, FDR and MDD) were divided into 2 independent subgroups (CT positive subgroup and CT negative subgroup). The CT negative subgroup consisted of individuals who either scored in the low or no category on the CTQ-SF.

\section{Instruments}

\section{Childhood Trauma Questionnaire-Short Form (CTQ-SF)}

The CTQ-SF is a 28-item self-report retrospective inventory intended to measure abuse and neglect of children ages 12 and older [29, 30]. The Chinese version used has been tested and shown with good reliability and validity in the general population [31, 32]. It is a 5-point Likert scale ranging from "Never true" to "Very often true". The CTQ-SF contains five subscales, which measure three types of abuse and two types of neglect: namely emotional abuse (EA), physical abuse (PA), sexual abuse (SA), emotional neglect (EN) and physical neglect (PN). The score for each scale is a sum of scores of specific items, and the total score of the CTQ-SF is a sum of scores on all scales. Severity of each trauma category based on cut-off scores was quantified as "none (or minimal)", "low (to moderate)", "moderate (to severe)" and "severe (to extreme)". In this study, cut-off scores for "moderate (to severe)" was employed to classify study 
participants as positive for history of specific trauma category. The CTQ cut-off scores for "moderate (to severe)" are as follows: $\mathrm{EA} \geq 13, \mathrm{PA} \geq 10, \mathrm{SA} \geq 8, \mathrm{EN} \geq 15$, and $\mathrm{PN} \geq 10$.

\section{Eysenck Personality Questionnaire (EPQ)}

The EPQ is a self-report questionnaire for measuring personality dimensions (traits), including a junior version (for 7-15 years old) and an adult version (for 16 years and older) [33]. The validated Chinese version of EPQ for adults has 88 questions and it has been tested to show good reliability and validity [34]. The EPQ consists of 4 personality dimensions (traits), namely extraversion/introversion (E), with a higher score meaning greater extroversion); neuroticism/stability $(\mathrm{N})$, with a higher score meaning less stable emotions; psychoticism/socialization $(\mathrm{P})$, with a higher score meaning higher psychoticism; lie/social desirability (L), with a higher score meaning higher tendency for dissimulation and fake on the responses. The score for each dimension is a sum of the responses ("agreement" scored 1, "disagreement" scored 0) to specific questions.

\section{Health Questionnaire (PHQ-9)}

Health Questionnaire (PHQ-9), a nine-item instrument, was purposed primarily for the application in primary care [35]. It was developed by referencing the diagnostic standard applied for the assessment of depression-induced disorder, as cited from the 4th edition of Diagnostic and Statistical Manual [36]. The involved items are subjected to ratings ranging from not at all to almost every day for the most recent two weeks with a four-point scale for duration. The PHQ-9 has been used widely for screening, diagnosis, monitoring treatment response.

\section{Statistical analysis}

Statistical analyses were conducted using the SPSS (version 19.0). All differences were considered statistically significant when $\mathrm{p}<0.05$ for both directions. The 
continuity-adjusted $\chi^{2}$ test and the Fisher's exact test were used to compare the distribution of categorical variables among the three groups and between groups with and without CT. The Kruskal-Wallis test was performed to compare age and education level of the three groups. The t-test and the Mann-Whitney test were performed to compare age, education level, E, N, P, L scores between three groups with and without CT. Pearson's correlation, spearman's correlation and multiple linear regression analysis were adopted to assess the association between personality traits and types of $\mathrm{CT}$ in FDR and other two groups.

\section{Results}

\section{Sociodemographic characteristics}

There were no significant differences in sex, age and education level between the three groups (Table 1). There was a significant difference in age $(\mathrm{P}=0.02)$ between those comparisons. People with CT had significantly higher N scores than those without CT 
Individuals with CT showed significantly lower L score than those without CT in MDD $(\mathrm{t}=2.331, \mathrm{p}<0.05)($ Table 2).

\section{Association between CT subtypes and personality dimensions}

In FDR, significantly positive correlations were found between N and SA, EN, PN and CTQ total $(\mathrm{r}=0.344-0.452, \mathrm{p}<0.05)$, and between P and CTQ $(\mathrm{r}=0.336, \mathrm{p}<0.05)$. Significantly negative correlations were found between L and EN, CTQ ( $r=-0.446$ - $0.375, \mathrm{p}<0.05)$ (Table 3).

In $\mathrm{HC}$, significantly positive correlations were found between $\mathrm{N}$ and $\mathrm{SA}$, as well as CTQ $(\mathrm{r}=0.290-0.368, \mathrm{p}<0.05)$; and between P and EA, PN, and CTQ ( $\mathrm{r}=0.292$ 0.303, $\mathrm{p}<0.05$ ). Significantly negative correlations were found between L and EA, SA, EN, PN, and CTQ $(r=-0.286--0.248, \mathrm{p}<0.05)$. In MDD, positive correlations were found between $\mathrm{N}$ and EA, PA, CTQ $(r=0.223-0.333, \mathrm{p}<0.05)$; P and EA, SA, CTQ $(r=0.240-0.356, p<0.05)$. Significant negative correlations were found between $E$ and EA, EN, PN, CTQ ( $\mathrm{r}=-0.397--0.246, \mathrm{p}<0.05)$; L and EA, CTQ ( $\mathrm{r}=-0.266--0.245$, $\mathrm{p}<0.05)$ (Appendix 1).

Multiple linear regression analyses of abuse and neglect scores on the EPQ subscale

A multiple linear regression model was used to adjust for co-variates. In FDR, EN contributed to a greater probability of $\mathrm{N}$ and $\mathrm{P}\left(\mathrm{R}^{2}=0.155-0.214, \mathrm{~F}=6.066-9.010\right.$, $\mathrm{p}=0.005-0.019) ; \mathrm{EN}$ was associated with a lower probability of $\mathrm{L}\left(\mathrm{R}^{2}=0.199, \mathrm{~F}=\right.$ 8.211, $\mathrm{p}=0.007)$ (Table 4).

In $\mathrm{HC}, \mathrm{SA}$ and EA contributed to a greater probability of $\mathrm{N}\left(\mathrm{R}^{2}=0.184, \mathrm{~F}=9.683\right.$, $\mathrm{p}<0.001)$; EA and $\mathrm{PN}$ were associated with a greater probability of $\mathrm{P}\left(\mathrm{R}^{2}=0.140, \mathrm{~F}=\right.$ 6.979, $\mathrm{p}=0.002)$; EA was associated with a lower probability of $\mathrm{L}\left(\mathrm{R}^{2}=0.113, \mathrm{~F}=\right.$ 11.109, $\mathrm{p}=0.001)$. In MDD, significant negative associations were found between $\mathrm{E}$ and 
$\mathrm{EA}\left(\mathrm{R}^{2}=0.158, \mathrm{~F}=15.549, \mathrm{p}<0.001\right) ; \mathrm{N}$ and $\mathrm{EA}, \mathrm{EN}\left(\mathrm{R}^{2}=0.162, \mathrm{~F}=7.950, \mathrm{p}=0.001\right)$; $\mathrm{P}$ and $\mathrm{EA}, \mathrm{SA}\left(\mathrm{R}^{2}=0.199, \mathrm{~F}=10.201, \mathrm{p}<0.001\right) ; \mathrm{L}$ and $\mathrm{EA}\left(\mathrm{R}^{2}=0.071, \mathrm{~F}=6.319\right.$, $\mathrm{p}=0.014)($ Appendix 2).

\section{Discussions}

This study was the first to verify the association between CT and personality traits in unaffected FDRs of patients with MDD. We found no significant differences between FDR and HC in personality traits. In FDRs, CT was associated with a higher score on neuroticism, psychoticism and lie, with the association with neuroticism the strongest. Besides, in terms of subtype, emotional neglect was the one to be found to have the strongest association with neuroticism.

Many previous studies have demonstrated that a high proportion of patients with severe depression have maladaptive personality traits. Studies have also shown that personality disorders at baseline in patients with MDD were robust predictors of a slow remission [37], and even after they achieved remission, personality disorders were a strong predictor of prospectively of accelerated relapse [37].

However, there have been scarce studies on personality traits in first-class relatives of patients with MDD and the effects of child abuse on personality traits. In contrast to the prior studies $[38,39]$, this study showed that compared with healthy controls; there were no significant differences on the EPQ scores in FDRs. Moreover, there is no relevance of depression (PHQ-9) to the personalities in first-degree relatives. Genetic and personality factors might be two relatively separate risk factors in the development of depression. 

aggregation in personality traits between patients with MDD and their unaffected FDRs.

As suggested by the existing studies on the relationships between personality [40], a definitive conclusion regarding the impact of heredity and environmental factors on personality has not yet been drawn in recent studies. Some researchers suggested that there was no close association between heredity and personality [41].

This study also found no significant differences in childhood trauma between FDRs and the healthy control group. On the other hand, studies have repeatedly shown the association between CT and risk of developing depression [42, 43]. Our findings may suggest that CT might involve a separate mechanism from hereditary factors in causing depression.

Furthermore, we found that among the FDRs, neuroticism, psychoticism and lie were significantly higher in the group with CT than those without CT, with neuroticism being the highest. In addition, CT was associated with all the four personality dimensions in MDD patients. This is the first study to show a significant between childhood trauma and personality dimensions in unaffected FDRs. Previous studies have shown the personality traits, especially neuroticism, may mediate the effects of CT on MDD [43].

Individuals with CT displayed significantly higher N scores than those without $\mathrm{CT}$ in FDRs. As reported by the prior studies [44, 45], CT was associated with higher $\mathrm{N}$ scores in both the general population and in patients with MDD. Our findings in the FDRs of patients with MDD provide new knowledge. Similar to the previous studies [15], our study showed personality traits were significantly associated with childhood trauma in FDR. E, N, P and L were primarily associated with EN among CT. The 
subtype of childhood trauma that were most associated with neuroticism was emotional neglect. Difference from our findings, a few other studies reported emotional abuse was the one with the strongest association with neuroticism in healthy controls (references here); and individual with avoidant personality disorder [42, 43]. It is worth noting, they also found that emotional neglect was associated with neuroticism, secondary to emotional abuse. Additionally, the associations between neglect and multiple personality dimensions have been reported in several prior studies in healthy controls [13]. It may be related to different sample characteristics and different independent variables included in the analysis.

Despite the absence of a full explanation of the neurobiological mechanism of changes to personality in response to $\mathrm{CT}$, some potential mechanisms have been proposed. People who sustained trauma in childhood tend to show a lower level of glucocorticoid expression, in addition to a range of distinct characteristics including changed methylation status in the neuron-specific glucocorticoid receptor promoter, long-lasting hypothalamic-pituitary-adrenal axis change and excessively active autonomic nervous system $[46,47]$. As indicated by the different cortisol levels among patients suffering with trauma-induced personality disorder, the processing was considered a potential cause to trigger the distinct coping mechanisms [48, 49].

\section{Limitations}

Several limitations of this study should be acknowledged. Data on CT were obtained using the childhood trauma Questionnaire-Short Form (CTQ-SF), which was a retrospective self-report questionnaire without independent authentication. Accordingly, the validity of reports might be affected by possible memory biases of the patients or excessive sensitivities to some CT experiences. Second, only a few risk factors were included, and the generalizability of the regression analyses should be 
considered with cautions. Finally, due to the small sample size of FDRs of patients with MDD, findings should be considered to be the preliminary.

\section{Conclusions}

Childhood trauma is associated with neuroticism, psychoticism and lie, and emotional neglect is strongly associated with neuroticism in first degree relatives of patients with MDD. The findings need to be replicated in a larger samples and the effects of the personality traits in first degree relatives of patients with MDD on mental health should also be studied further.

\section{Reference}

1. Bienvenu OJ, Brown C, Samuels JF, et al. Normal personality traits and comorbidity among phobic, panic and major depressive disorders. Psychiatry Res, 2001;102(1):73-85.

2. Outlaw J, Wilborn C, Smith A, et al. Effects of ingestion of a commercially available thermogenic dietary supplement on resting energy expenditure, mood state and cardiovascular measures. J Int Soc Sports Nutr, 2013;10(1):25.

3. Hirschfeld RM, Klerman GL. Personality attributes and affective disorders. Am J Psychiatry, $1979 ; 136(1): 67-70$.

4. Schapira K, Roth M, Kerr T, Gurney C. The prognosis of affective disorders: the differentiation of anxiety states from depressive illnesses. Br J Psychiatry, 1972;121(561):175-81.

5. Duggan CF, Lee AS, Murray RM. Does personality predict long-term outcome in depression? $\mathrm{Br} \mathrm{J}$ Psychiatry, 1990;157(1):19-24.

6. Quilty LC, De Fruyt F, Rolland JP, Kennedy SH, Rouillon PF, Bagby RM. Dimensional personality traits and treatment outcome in patients with major depressive disorder. J Affect Disord, 2008;108(3):241-50.

7. Weissman MM, Prusoff BA, Klerman GL. Personality and the prediction of long-term outcome of depression. Am J Psychiatry, 1978;135(7):797-800.

8. Kendler KS, Gatz M, Gardner CO, Pedersen NL. Personality and major depression: A Swedish longitudinal, population-based twin study. Arch Gen Psychiatry, 2006;63(10):1113-20. 
9. Boyce P, Parker G, Barnett B, Cooney M, Smith F. Personality as a vulnerability factor to depression. Br J Psychiatry, 1991;159(1):106-14.

10. Klein DN, Kotov R, Bufferd SJ. Personality and depression: Explanatory models and review of the evidence. Annu Rev Clin Psychol, 2011;7:269-95.

11. Klein DN. Depressive personality in the relatives of outpatients with dysthymic disorder and episodic major depressive disorder and normal controls. J Affect Disord, 1999;55(1):19-27.

12. Wu PJ, Chang SM, Lu MK, et al. The profile and familiality of personality traits in mood disorder families. J Affect Disord, 2007;98(3):367-74.

13. de Carvalho HW, Pereira R, Frozi J, Bisol LW, Ottoni GL, Lara DR. Childhood trauma is associated with maladaptive personality traits. Child Abuse Negl, 2015;44:18-25.

14. Hengartner MP, Cohen LJ, Rodgers S, Müller M, Rössler W, Ajdacic-Gross V. Association between childhood maltreatment and normal adult personality traits: Exploration of an understudied field. Pers Disord, 2015;29(1):1-14.

15. Li XB, Wang ZM, Hou YZ, Wang Yi, Liu JT, Wang CY. Effects of childhood trauma on personality in a sample of Chinese adolescents. Child Abuse Negl, 2014;38(4):788-96.

16. Vickers NJ. Animal communication: when i'm calling you, will you answer too? Curr Bio, 2017;27(14):R713-R5.

17. Li W, Meng X, Xu Z, et al. Prevalence, correlates of major depression: A mental health survey among undergraduates at a mainland C hinese university. Asia-Pac Psychiat, 2016;8(3):206-14.

18. Bernet CZ, Stein MB. Relationship of childhood maltreatment to the onset and course of major depression in adulthood. Depress Anxiety, 1999;9(4):169-74.

19. Nelson J, Klumparendt A, Doebler P, Ehring T. Childhood maltreatment and characteristics of adult depression: meta-analysis. Brit J Psychiat, 2017;210(2):96-104.

20. Tunnard C, Rane LJ, Wooderson SC, et al. The impact of childhood adversity on suicidality and clinical course in treatment-resistant depression. J Affect Disorders, 2014;152:122-30.

21. Hayashi Y, Okamoto Y, Takagaki K, et al. Direct and indirect influences of childhood abuse on depression symptoms in patients with major depressive disorder. BMC Psychiatry, 2015;15(1):244.

22. Toda $\mathrm{H}$, Inoue $\mathrm{T}$, Tsunoda $\mathrm{T}$, et al. Affective temperaments play an important role in the relationship between childhood abuse and depressive symptoms in major depressive disorder. Psychiatry Res, 
23. Gamble SA, Talbot NL, Duberstein PR, et al. Childhood sexual abuse and depressive symptom severity: the role of neuroticism. J Nerv Ment Dis, 2006;194(5):382-5.

24. Lee MA, Song R. Childhood abuse, personality traits, and depressive symptoms in adulthood. Child Abuse Negl, 2017;65:194-203.

25. Nakai Y, Inoue T, Toda H, et al. The influence of childhood abuse, adult stressful life events and 2014;158:101-7.

26. Ono K, Takaesu Y, Nakai Y, et al. Associations among depressive symptoms, childhood abuse, neuroticism, and adult stressful life events in the general adult population. Neuropsychiatr Dis Treat, 2017; 13:477-82.

27. Hovens JG, Giltay EJ, van Hemert AM, Penninx BW. Childhood maltreatment and the course of depressive and anxiety disorders: the contribution of personality characteristics. Depress Anxiety, 2016;33(1):27-34.

28. First MB, Gibbon M. Structured clinical interview for DSM-IV-TR axis I disorders, research version, patient edition (SCID-I/P). New York: Biometrics Research, 2002.

29. Bernstein DP, Fink L. Childhood trauma questionnaire: A retrospective self-report: Manual. Toronto and Orlando: Harcourt Brace \& Company, 1998.

30. Bernstein DP, Stein JA, Newcomb MD, et al. Development and validation of a brief screening version of the Childhood Trauma Questionnaire. Child Abuse Negl, 2003;27(2):169-90.

31. Fu W, Yao S, Yu H, et al. Initial reliability and validity of Childhood Trauma Questionnaire (CTQSF) applied in Chinese college students. Chin J Clin Psychol, 2005;13(1):40-2.

32. Zhao X, Zhang Y, Longfei L, Zhou Y. Evaluation on reliability and validity of Chinese version of childhood trauma questionnaire. Chin J Tissue Eng Res, 2005;9(16):209-11.

33. Eysenck HJ, Eysenck SBG. Manual of the Eysenck Personality Questionnaire (junior and adult). London: Hodder and Stoughton, 1975.

34. Yaoxian G. Eysenck personality questionnaire revised in China. Psychol Sci, 1984;4:11-8.

35. Kroenke K, Spitzer RL, Williams JB. The PHQ-9: Validity of a brief depression severity measure. J Gen Intern Med, 2001;16(9):606-13. 
36. American Psychiatric Association. Practice guideline for the treatment of patients with panic disorders. Am J Psychiatry, 1998;159(4).

37. Grilo CM, Stout RL, Markowitz JC, et al. Personality disorders predict relapse after remission from an episode of major depressive disorder: A six-year prospective study. J Clin Psychiatry, 2010;71(12):1629.

38. Ouimette PC, Klein DN, Pepper CM. Personality traits in the first degree relatives of outpatients with depressive disorders. J Affect Disord, 1996;39(1):43-53.

39. Klein E, Kreinin I, Chistyakov A, et al. Therapeutic efficacy of right prefrontal slow repetitive transcranial magnetic stimulation in major depression: a double-blind controlled study. Arch Gen Psychiatry, 1999;56(4):315-20.

40. Coid B. Genes and environment in personality development. Crim Behav Ment Health, 1995;51(1):59-60.

41. Eysenck HJ. Genetic and environmental contributions to individual differences: The three major dimensions of personality. J Pers, 1990;58(1):245-61.

42. Klein JP, Roniger A, Schweiger U, Späth C, Brodbeck J. The association of childhood trauma and personality disorders with chronic depression: A cross-sectional study in depressed outpatients. J Clin Psychiatry, 2015;76(6):e794.

43. Zhang M, Han J, Shi J, et al. Personality traits as possible mediators in the relationship between childhood trauma and depressive symptoms in Chinese adolescents. J Psychiatr Res, 2018;103:1505.

44. Allen B, Lauterbach D. Personality characteristics of adult survivors of childhood trauma. J Trauma Stress, 2007;20(4):587-95.

45. Wang Y, Yang JL, Jiao QY. Childhood abuse impact on clinical and personality characters among anxiety associated depressive patients. Chin J Behav Med Brain Sci, 2015;24(5):451-4.

46. Heim C, Newport DJ, Heit S, et al. Pituitary-adrenal and autonomic responses to stress in women after sexual and physical abuse in childhood. JAMA, 2000;284(5):592-7.

47. Weaver IC, Cervoni N, Champagne FA, et al. Epigenetic programming by maternal behavior. Nat Neurosci, 2004;7(8):847-54.

48. Oquendo M, Echavarria G, Galfalvy H, et al. Lower cortisol levels in depressed patients with 
comorbid post-traumatic stress disorder. Neuropsychopharmacol, 2003;28(3):591.

447

49. Volckaert FA, Hellemans B, Batargias C, et al. Heritability of cortisol response to confinement stress in European sea bass dicentrarchus labrax. Genet Sel Evol, 2012;44(1):15.

449 


\section{Supplementary Files}

This is a list of supplementary files associated with this preprint. Click to download.

- Appendix.docx 